\title{
Reflection on the Spread of TCM Under the Belt and Road Initiative Background
}

\author{
Zhongkai Cao \\ School of Foreign Languages \\ Hubei University of TCM \\ Wuhan City, Hubei Province, 430065, PRC \\ caozk2002@163.com
}

\author{
Fen Huang* \\ Department of Integrated Chinese and Western Medicine, \\ Tianyou Hospital Affiliated to Wuhan University of Science \\ and Technology, \\ Wuhan City, Hubei Province, 430061, PRC \\ Huangf3002@163.com
}

\author{
Hongying Yang \\ School of Foreign Languages \\ Hubei University of TCM \\ Wuhan City, Hubei Province, 430065, PRC
}

\begin{abstract}
A wider spreading and larger scale of exchange of Traditional Chinese Medicine (TCM) along the Belt and Road Initiative (BRI) countries would significantly contribute to the advancement of public health in the world. However, due to the differences in the medical care system and cultural background, the spreading of TCM faces big challenges. Based on the analysis of acceptance of TCM along with BRI countries, it is proposed in this article to optimize bilateral cooperation mechanism, enhance people-to-people communication, and strengthen the innovation of TCM.
\end{abstract}

Keywords-TCM spreading; Belt and Road Initiative background; challenges; solutions

\section{INTRODUCTION}

Since the launch of Belt and Road Initiative, the Chinese government keeps on promoting traditional Chinese medicine to help bring a healthy life to people along the route. In 2016, President Xi further proposed in Uzbekistan to deepen medical cooperation over disease control, traditional medical care, and work together to build "a Silk Road of health". This has greatly promoted the in-depth external communication and exchange of traditional Chinese medicine, and contributes to enhance the influence of TCM along the Belt and Road countries and regions. [1]

However, there are still many challenges in the spread and exchange of TCM. To address the difficulties, people in this field must carry out in-depth research and analysis in regard of the status quo about TCM along the BRI route, and then go deeper to look for workable solutions. Thus, this research presents a detailed description of the current spreading of TCM along the Belt and Road countries, and then made a list of the obstacles involved. On such a basis, this article, at last, put forward some suggestions to help better promote the spreading of TCM.

\section{THE SPREADING OF TCM ALONG BRI COUNTRIES}

The countries along the Belt and Road route have a long history of using traditional Chinese medicine or traditional medicine, and they are rich in local traditional medical resources. In recent years, with the flourishing of Chinese medicine trade, traditional medicine has become a new economic growth point in many countries. With the implementation of the Belt and Road strategy, China and countries along the route have broader prospects for exchanges and cooperation in the field of traditional Chinese medicine.

\section{A. The Spreading of TCM in South Asia}

Traditional Chinese medicine has a long history of development in Singapore, and this country also serves as an ideal base for the spreading of TCM in Southeast Asia. There are more than 30 TCM institutions and more than 1,000 TCM clinics in the city state. The Singapore Ministry of Health has established the Chinese Medicine Administration and the Singapore Chinese Medicine Coordinating Committee to further strengthen the management of TCM. [2]

In Malaysia, another important southeast Asian country, there are more than 3,000 drug stores selling TCM products and over 800 members in the Chinese Medicine Physician Union, most of whom are conducting medical practices related with TCM. The Malaysian Ministry of Health has always been very concerned and supportive of TCM, but has not yet accepted the registration of Chinese medicine practitioners.

The Thai government recognizes the legal status of TCM and only issues temporary licenses for TCM practitioners who pass the examination. As early as in 1987, the government officially approved the Chinese Herbal Medicine Bill. There are more than 800 Chinese medicine stores, 10 large Chinese medicine wholesalers, and about 40 small and medium-sized TCM enterprises in this country. 
Vietnam has long proposed the combination of Chinese medicine and Western medicine. There are nearly 200 largescale TCM stores, small and medium-sized TCM pharmacies are widespread in the country. Over 180 kinds of Chinese herbal medicines are exported from China to Vietnam each year. At present, a total of 16 TCM pharmaceutical manufacturers in China have obtained operating licenses in Vietnam.

It can be concluded from above that since Southeast Asian countries are not only geographically adjacent to China, but also closely linked culturally. Thus, the spread of traditional Chinese medicine in Southeast Asian countries is generally supported by governments and people in these countries.

\section{B. The Spreading of TCM in Western Europe}

Traditional Chinese medicine can be found in more than 20 west European countries along the BRI route, of which its level of acceptance in Germany, France, and UK attracts more attention.

Germany is the country that uses most Chinese herbal medicines in western European countries, and more than 58\% of Germans take Chinese herbal medicine. Chinese herbal medicines are available at any pharmacy in Germany, and the annual sales of ginkgo preparations in Germany even exceed $\$ 100$ million, besides, TCM has been also included in the botanical drug system at the Quetzut Institute.

France is the second largest market for traditional Chinese medicine in the European Union. In 1952, the French Academy of Medical Sciences recognized that acupuncture is a scientific medical practice, in 1985; it decided to include the education of TCM in the curriculum of medical universities. There are 23,000 pharmacies in the market, of which more than 10,000 are the main sales channels for herbal and natural Chinese medicines.

The UK herbal market is estimated to be 65 million pounds and is the third largest market for TCM in the European Union. Fish oil, which is used for heart protection, is the most widely sold TCM product in the UK market. Other widespread TCM products include painkillers, cough medicines and skin diseases. There are more than 1200 TCM pharmacies in the UK, and many of them are major channels for Chinese medicine sales. Moreover, a majority of the community hospitals in British cities now use a variety of herbal medicines to treat the disease. [3]

The three Western European countries above are referred to as the major market of TCM products in EU. In fact, Chinese medicine also has a fast development in Czech, Italy and European countries. In general, due to cultural differences between China and the West, there is still much room for improvement in the spreading of Chinese medicine in Western Europe.

\section{The Spreading of TCM in Africa}

The spreading of TCM on the African continent does not have a long history, but the growth is rather steady. In the 1950s, China sent the first batch of medical aid teams to Africa, during which process TCM experts played significant roles in tackling the infectious disease. In the past 60 years, TCM medical treatment has been officially incorporated into national medical systems in countries such as in South Africa, Tanzania, etc. Acupuncturists have been officially licensed in many African countries too, many typical TCM products such as artemisinin has already saved millions of lives in this area. Up to today, the total import and export of Chinese medicine between China and Africa reached 80 million dollars, more than 2,000 TCM practitioners have been sent to Africa, and hundreds of TCM pharmaceutical companies have also set up branches in Africa to provide Chinese medicine services to Africans. [4]

\section{The ChAlLENGES IN THE SPREADING OF TCM}

The spreading of TCM along BRI countries is by no means a smooth process, by gathering and analyzing the information of medical policies and TCM related reports in these countries, the researchers summed up 3 major obstacles in the process of TCM spreading.

\section{A. Medical Policy Limitation and Trade Barrier}

There are over 100 countries along the Belt and Road route, and the legislation about TCM varies in each country and region, the acceptance of Chinese medicine by the general public is also not at the same level. In general, Southeast Asian countries have a higher acceptance of TCM from the government to the public. The legislation and management related to TCM are generally not as comprehensive in other countries and regions, and it even remains blank in many countries. The safety of TCM is always taken as an excuse for legal restrictions in Europe. [5]

So far, countries along the Belt and Road route have not yet had a unified, specialized international standard and norms related to TCM. Since modern western medicine is now taken as the international standard medical system, the related production, testing and evaluation of traditional Chinese medicine are often judged according to the quantitative criteria of Western medicine in most counties. Therefore, the registration, market access to TCM products often lack specialized guidance.

In such a context as mentioned above, the exchange and cooperation over TCM in the countries along the BRI route meets enormous trade barriers. In recent years, even though many policymakers in these countries have actively introduced TCM to meet the health and medical needs of their citizens. On the other hand, in order to safeguard their interests in medical industry, they often hesitant to provide legislative protection for TCM. Influenced by the legal and technical restrictions on TCM, the international trade of TCM gets much dampened.

\section{B. Fundamental Differences between Chinese Medicine and Western Medicine}

At present, Western modern medicine dominates the world medical system, and is regarded as an internationally recognized medical standard. Traditional Chinese medicine is often considered as an alternative medicine to the mainstream medical system of Western medicine. Western medicine 
emphasizes more of the logical reasoning and is developed on the basis of experimental research. Based on the ancient Chinese philosophy of simple materialism and thoughts of harmony, traditional Chinese medicine then established its own unique medical theory by interacting with ancient Chinese astronomy, meteorology, geography and many other disciplines. The Chinese and Western culture serves respectively as the root of Chinese and Western medicine, however, people with the western culture background often fail to realize such a fact, their belief of cultural superiority even leads them to believe that western medicine is the only one medical approach in the world. Traditional Chinese medicine is then regarded as unscientific, unacceptable and even witchcraft.

\section{Lack of Innovation in TCM}

As both a medical approach and a carrier of Chinese culture, traditional Chinese medicine needs to be inherited and innovated at the same time, since innovation is the driving force to keep things update. At present, there is a clear shortage of theoretical foundation in regard of TCM medical treatment. Due to differences in history, cultural background and mode of thinking, Chinese medicine and modern medicine have significantly different characteristics, the scientific connotation of TCM theory should not be expressed solely from the perspective. On the meantime, the progress of modern civilization also rejects the interpretation of TCM from the old-fashioned and even obsolete manner, thus demanding an urgent innovation of TCM theories to meet the public needs. Moreover, the technical principles such as the method of diagnosis, syndrome differentiation and the mode of administration of TCM have not undergone accurate evaluation or been widely applied in modern scientific researches. The working mechanism of many TCM therapies are still unclear, lacking of support from modern science. In short, there still remains a big gap between the theoretical and technical system of TCM and western medicine, which requires more devotion and efforts of TCM practitioners to further the innovation.

\section{SUGGESTIONS FOR THE SPREADING OF TCM}

\section{A. Enhancing the High-Level Cooperation}

It is high time for people in the TCM industry to fully explore the potential of the existing cooperation mechanism between the governments, actively promote the implementation of the signed bilateral cooperation agreement on TCM, and the governmental organizations need to continue carrying out high-level exchanges between Chinese and foreign governments and international organizations. It is necessary for policymakers in China to build up intergovernmental consultation and coordination mechanism, which helps to strengthen contacts between senior officials of both sides, expands cooperation with governments on making policies and regulations regarding TCM market access and supervision. In this way, people will be able to coordinate and solve major problems from a global perspective, and create a favorable policy environment for TCM to spread along the Belt and Road countries. [6]
In this field, the Chinese government gets a bigger role to play by strengthening the protection of the time-honored brand of TCM, promoting TCM products and services as a demonstration of intangible cultural heritage. They can also offer policy guidance and financial funding for TCM education and cultural exchange programs in countries along the BRI route. Besides, through the coordination of government departments and cooperation with international organizations, TCM practitioners could build international TCM information centers to better share and exchange medical information along the Belt and Road Initiative countries.

\section{B. Encouraging People to People Exchange}

TCM organizations and practitioners need to take advantage of the Confucius Institute to spread Chinese medicine culture. At present, there are more than 400 Confucius Institutes overseas, by integrating Chinese medicine culture, traditional Chinese medicine science into the teaching of Chinese, people can not only enrich the teaching content, but also provide a more comprehensive interpretation of TCM culture. TCM experts in this process can play an important role by giving lectures, offering medical consultations, and holding academic communication conferences with western counterparts. Such kind of cultural exchange activities in the Confucius Institute will contribute a lot to expand the influence of TCM culture through lectures and free clinical services.

Meanwhile, it is also necessary to support qualified colleges and universities to set up communication centers for TCM along with the Belt and Road countries, and jointly cultivate local talents who will master knowledge of both Chinese medicine and local medicine. Considering the popularity and tremendous power of online education, an international English online course of TCM jointed sponsored by multiple universities needs to be established to attract more foreign audiences and students who are interested in Chinese medicine. Scholarships and funds also need to be set up to sponsor such groups of TCM fans to further the learning and experiencing of TCM in China.

\section{Promoting the Innovation of TCM}

At present, the slowing down of TCM spreading can be partly attributed to the limited access of Chinese medical knowledge, degradation of the quality of TCM products, and the ongoing marginalization of the status of TCM in the modern medical system. "Blacksmith needs its own hardware", TCM practitioners thus should spare no efforts to seek for self-improvement, self-innovation of TCM knowledge, so as to play a leading role in the innovative development of Chinese medicine. People also need to deepen the basic theoretical research of Chinese medicine, establishing a theoretical system with clear concepts and reasonable structure. Considering the big advantage of TCM in the treatment of infectious disease, frequently-occurring diseases, chronic diseases, and in disease prevention, TCM companies can invest more on pharmaceutical researches and product design, so as to come up with a number of branded TCM products and services to cater to the local market needs. The governmental 
organizations can make innovations to speed up the legislation of laws concerning TCM, implementing strict supervision of TCM products and services, and help to create an environment where TCM could not just survive but flourish.

\section{CONCLUSION}

This article starts from exploring the current spreading of TCM along with the Belt and Road countries, then points out some obstacles encountered and finally puts forward some suggestions to promote its development. Traditional Chinese medicine contains unique philosophical thinking and values of Chinese civilization, which is also the eternal charm of it. The Belt and Road Initiative offers a golden opportunity for the spreading, exchanging and self-renewing of TCM and the Chinese culture it embodies. By addressing the challenges mentioned above, the stage of TCM will get larger and larger, and the role of TCM in international health care and disease prevention will be significantly enhanced too.

\section{ACKNOWLEDGMENT}

This research is financially supported by the "Qingmiao" academic fund of science and technology department of Hubei University of Chinese Medicine, under the program "Translation and external spreading of Bencao Gangmu
(5112-00070201)”. Sincere thanks also go to colleagues from the Department of Foreign Languages of Hubei University of Chinese Medicine for their constructive suggestions in editing the research paper.

\section{REFERENCES}

[1] Xi Jinping, Joint Efforts for Shared Community, A Collection of Open Speech, Bejing: People’s Press, 2017, pp.107-114. (In Chinese)

[2] Shen Junlong, Wangrui, Exploring the Spreading of TCM in Southeast Asia. Beijing: Social Sciences Academic Press, 2017, pp.52-183. (In Chinese)

[3] Man Fongmei, Various medical specialist prominently occurs and s hundred schools of thought contend: current situation and prospect of traditional Chinese medicine in UK [J], Tianjin Journal of Traditional Chinese Medicine, 2010, pp.25-30.

[4] Cem Nalbantoglu. One Belt One Road Initiative: New Route on China's Change of Course to Growth [J], Open Journal of Social Science, 2017, 3(2).

[5] Tony McEnery, Qian Yufang. A corpus-based discourse study of Chinese medicine in UK national newspapers [J], Zhejiang University of Media and Communications, 2017, 8(1).

[6] Su Zheqian, Proceedings of 2015 5th International Conference on Applied Social Science. Singapore: Singapore Management and Sports Science Institute, pp. 102-138. 